Channel Influence for the Analysis of Interferences Between Automotive Radars

Lizette Lorraine Tovar Torres, Maximilian Steiner, Christian Waldschmidt 


\title{
Channel Influence for the Analysis of Interferences Between Automotive Radars
}

\author{
Lizette Lorraine Tovar Torres, Maximilian Steiner, Christian Waldschmidt \\ Institute of Microwave Engineering, Ulm University, 89081 Ulm, Germany
}

\begin{abstract}
This paper presents the channel influence when simulating interference between automotive radars in realistic scenarios. As a starting point, a simple approach considering only the line-of-sight (LoS) component of the signal transmitted by the interferer radar is presented. Following, the influence of the ground reflections in interference situations is analyzed by introducing the two-path model. Properties of the road surface such as roughness and the Fresnel reflection coefficient are considered in order to obtain a more realistic simulation. A sweep of all the possible ranges between victim and interferer radars is performed, and the influence of the interferer radar is analyzed. Finally, a statistical analysis of the key radar parameters is carried out in order to evaluate the interference behavior in the presented model.
\end{abstract}

Keywords - Automotive radar, interference, simulation.

\section{INTRODUCTION}

Modern vehicles are equipped with several radar sensors in order to provide driver assistance functions [1]. When considering real traffic scenarios, the problem of interference between automotive radars becomes a topic of interest making important to study the interference effects and to formulate counteracting measurements [2]. As part of an interference simulator developed in Matlab, this paper analyzes the interference issue by presenting two models, i.e. the line-of-sight model and the two-path model.

Current automotive radar sensors use the well-known chirp-sequence modulation scheme. Considering this kind of modulation, interference occurs when overlaps in frequency, time, and field of view (FoV) of two different sensors take place. Fig. 1 shows the key parameters of the chirp-sequence modulation scheme as well as a common interference situation. The influence of the interference is directly related to the receiver bandwidth of the victim radar $B_{\mathrm{RX}}$ and the difference between the slopes $\Delta \mu$ of the victim and interferer sensors. Previous works showed that when $\Delta \mu \neq 0$, the frequency ramps of victim and interferer cross each other, leading to an increased noise floor [3]. Consequently, targets with low radar cross section could not be detected anymore. A larger $B_{\mathrm{RX}}$ results in more samples affected with a considerable amount of interference power. On the other hand, $\Delta \mu=0$ means that victim and interferer frequency ramps are in parallel, leading to the appearance of ghost targets. Nevertheless, the last case is very unlikely to occur [3] and is not considered in this work.

\section{LINE-OF-Sight MODEL}

Consider two radars which operate at the same time, with overlapping frequency band, and located in such a way that the interferer is transmitting in the direction of the victim and

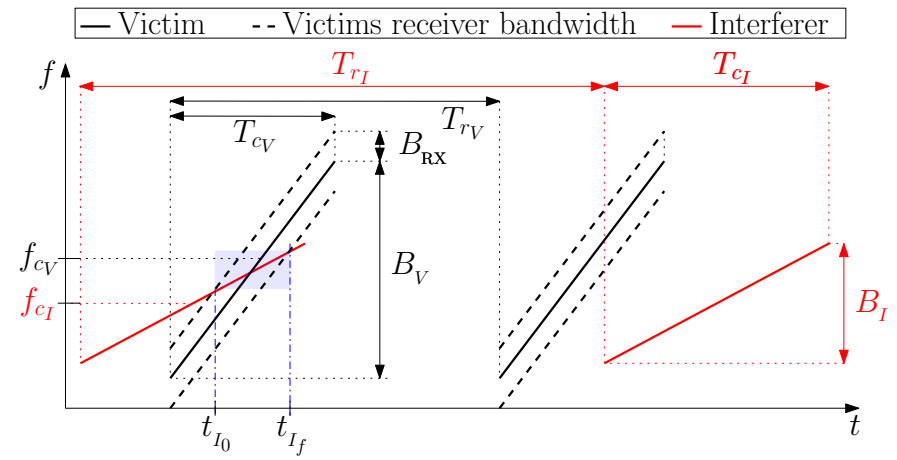

Fig. 1. Transmitted victim chirps (black) and interferer chirps (red). The interference is observed in the first victim chirp within the interval $\left[t_{I_{0}}, t_{I_{f}}\right]$, while in the second chirp no interference is present.

impinging within the victims FoV. For the following analysis the subscript " $V$ " refers to the victim sensor parameters and " $I$ " to the interferer sensor parameters. The phase of the signal generated by the local oscillator in the victim radar for one chirp can be described as

$$
\varphi_{\mathrm{TX}}(t)=2 \pi\left(f_{c_{V}} t+\frac{\mu_{V}}{2} t^{2}\right),
$$

where $f_{c}$ is the carrier frequency and $\mu=\frac{B}{T_{c}}$ the chirp slope.

The local oscillator in the interferer radar also generates a signal with a phase similar to (1). This signal impinges the victim radar with a delay $\tau_{I}=\frac{R}{c}+\frac{v}{c} t$, where $R$ and $v$ are the range and relative radial velocity between the victim and the interferer, respectively. For simplification in the further analysis, $v=0 \frac{\mathrm{m}}{\mathrm{s}}$ is considered. The phase of the received signal due to the interferer is then

$$
\varphi_{\mathrm{RX}}(t)=2 \pi\left(f_{c_{I}}\left(t-\tau_{I}\right)+\frac{\mu_{I}}{2}\left(t-\tau_{I}\right)^{2}\right) .
$$

After mixing and down conversion, the phase of the intermediate frequency signal due to the interferer is

$$
\begin{array}{r}
\varphi_{\mathrm{IF}}(t)=2 \pi\left(\frac{\Delta \mu}{2} t^{2}+\left(\Delta f_{c}-\mu_{I} \frac{R}{c}\right) t\right. \\
\left.-f_{c_{I}} \frac{R}{c}+\frac{\mu_{I}}{2}\left(\frac{R}{c}\right)^{2}\right),
\end{array}
$$

where $\Delta f_{c}$ and $\Delta \mu$ are the carrier frequency and slope differences of the involved radars, respectively, and $t \in\left[t_{I_{0}}, t_{I_{f}}\right]$.

The amplitude of the intermediate frequency signal is

$$
A \propto \frac{\sqrt{2}}{2} \sqrt{\frac{P_{I} G_{I} G_{V} \lambda^{2}}{(4 \pi R)^{2}}} \Gamma,
$$




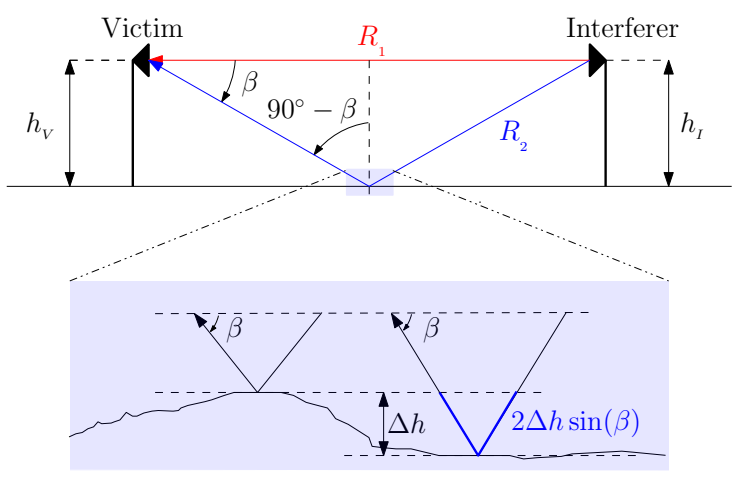

Fig. 2. Geometry of two-path model. Small fluctuations $\Delta h$ take place when the surface is not completely smooth. This produces path differences.

where $P_{I}$ and $G_{I}$ are the power and gain of the interferer antenna, and $G_{V}$ is the gain of the victim antenna. $G_{I}$ depends on the azimuth and elevation angle where the victim radar is seen by the interferer radar. Similarly, $G_{V}$ depends on the azimuth and elevation angle where the interferer is seen by the victim. The wavelength $\lambda$ is related to the carrier frequency of the victim radar. Road characteristics are included in the attenuation factor $\Gamma$, which, according to [4], can be defined as the product of the Fresnel reflection coefficient $\Gamma_{F}$ and a roughens factor $\Gamma_{R}$.

Denoting $R_{1}$ as the distance between the radars in the LoS, and assuming that the radars are located at the same height above the ground, the down-converted LoS signal component $s_{\mathrm{IF}_{1}}(t)$ has a phase $\varphi_{\mathrm{IF}_{1}}(t)$ defined by (3) with range $R=R_{1}$. The amplitude $A_{1}$ is given by (4), with $R=R_{1}$ and $\Gamma=1$. It is worth mentioning that, since the sensors are located at the same height, an elevation angle $\beta=0^{\circ}$ is considered when determining $G_{I}$ and $G_{V}$.

\section{TWO-PATH MODEL}

The two-path geometry is depicted in Fig. 2. The received signal due to the ground reflection $s_{\mathrm{IF}_{2}}(t)$ is an additional interferer signal with a phase $\varphi_{\mathrm{IF}_{2}}(t)$ defined also by (3) but with range $R=R_{2}$. Assuming that the radars are located at the same height $h_{V}$ above ground, then $R_{2}=\sqrt{\left(2 h_{V}\right)^{2}+R_{1}^{2}}$ and $\Delta R=R_{2}-R_{1}$. Moreover, the amplitude $A_{2}$ of $s_{\mathrm{IF}_{2}}(t)$ is defined by (4) with $R=R_{2}$, but now taking into account that the signal is impinging with an elevation angle $\beta \neq 0^{\circ}$. In this case, the attenuation factor $\Gamma=\Gamma_{F} \Gamma_{R} \neq 1$ must be studied in detail.

\section{A. Fresnel Reflection Coefficient}

Considering a plane surface, the amount of the reflected electric field at the interface can be defined using the Fresnel reflection coefficient. It mainly depends on the surface material properties at the operation frequency, the polarization, and the incident angle of the wave at the interface.

Current automotive radar sensors operate mainly in the $77 \mathrm{GHz}-81 \mathrm{GHz}$ frequency range and use vertical or horizontal polarization. Consequently, the reflection properties of asphalt at $77 \mathrm{GHz}$ are studied. The Fresnel reflection

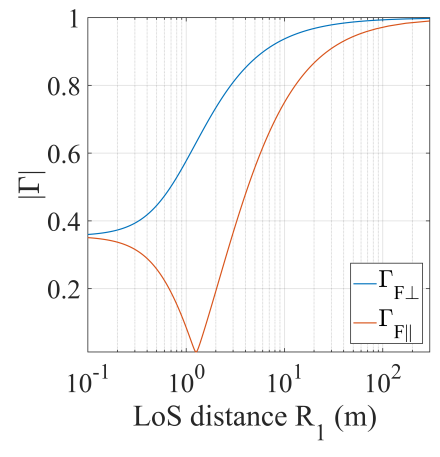

(a)

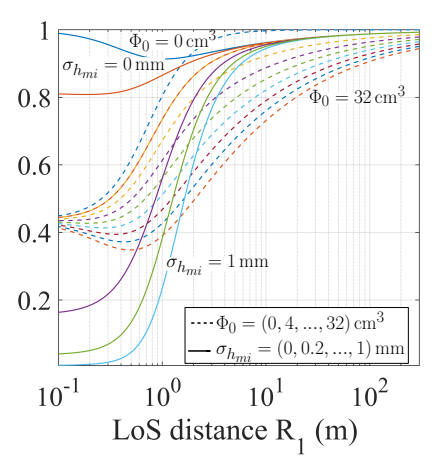

(b)
Fig. 3. The attenuation factor of a road surface is the product of the Fresnel reflection coefficient and the roughness coefficient: (a) Fresnel reflection coefficient $\Gamma_{F}$ of asphalt for vertically (red) and horizontally (blue) polarized radars at $77 \mathrm{GHz}$; (b) roughness coefficient $\Gamma_{R}$ for fixed $\sigma_{h_{m i}}=0.4 \mathrm{~mm}$ with $\Phi_{0}=(0,4, \ldots, 32) \mathrm{cm}^{3}$ in dotted lines, and for fixed $\Phi_{0}=0.4 \mathrm{~cm}^{3}$ with $\sigma_{h_{m i}}=(0,0.2, \ldots, 2) \mathrm{mm}$ in solid lines.

coefficient for vertically and horizontally polarized radars is defined in [4]. For the LoS case, the signal travels directly from the interferer to the victim and no reflection occurs. As a result, $\beta=0^{\circ}$ and $\left|\Gamma_{F_{\|}}\right|=\left|\Gamma_{F_{\perp}}\right|=1$.

According to [4], the mean value of the permittivity of dry asphalt at $77 \mathrm{GHz}$ is $\varepsilon_{r a}=4.4-\mathrm{j} 0.3$. Fig. 3a shows the absolute value of the Fresnel reflection coefficient of asphalt at $77 \mathrm{GHz}$ as a function of the LoS distance $R_{1}$ for vertically and horizontally polarized radars. It is observed that for short distances between the victim and the interferer sensors the interferer signal component $s_{\mathrm{IF}_{2}}(t)$ has a lower impact. For distances larger than $10 \mathrm{~m}$ the reflection coefficient increases and approaches to 1 . Besides, it can be seen that for vertically polarized radars a lower reflection coefficient is obtained, meaning a reduced effect of the interferer.

\section{B. Roughness Coefficient}

Since road surfaces are not completely smooth, part of the incident field is scattered in other directions reducing the specularly reflected components. Roughness can be understood as height fluctuations $\Delta h$ over a surface, as illustrated in Fig. 2, which cause phase differences $\Delta \varphi_{\Gamma_{R}}=\frac{2 \pi}{\lambda}(2 \Delta h \sin (\beta))$. Assuming Gaussian distributed height profiles, $\Delta h$ can be replaced by $\sigma_{h}$, the standard deviation of the profile height fluctuations inside the first Fresnel zone. Thereby, the roughness coefficient can be defined as [4]:

$$
\Gamma_{R}=\mathrm{e}^{-2\left(\frac{2 \pi}{\lambda} \sigma_{h} \sin (\beta)\right)^{2}} \text { with } \sigma_{h}=\sqrt{\sigma_{h_{m i}}^{2}+\sigma_{h_{m a}}^{2}} .
$$

Owing to the large extension of the first Fresnel zone, the standard deviation of the profile height fluctuations $\sigma_{h}$ is not only given by the micro-roughness of the material structure $\sigma_{h_{m i}}$ but also by the macro-roughness $\sigma_{h_{m a}}$, which describes the statistical unevenness due to road bumps. According to [4], $\sigma_{h_{m i}}<1 \mathrm{~mm}$, while the value for the macro-roughness is given by $\sigma_{h_{m a}}=\left(\Phi_{0} \sqrt{\lambda R_{1}} /(2 \pi \beta)\right)^{1 / 2}$. The spectral unevenness parameter $\Phi_{0}$ can vary from $2 \mathrm{~cm}^{3}$ 
to $8 \mathrm{~cm}^{3}$ in case of smooth asphalt, and in the extreme case of an unpaved road it could be larger than $128 \mathrm{~cm}^{3}$. Fig. $3 \mathrm{~b}$ depicts the roughness coefficient defined in (5) for good asphalt $\left(\sigma_{h_{m i}}=0.4 \mathrm{~mm}, \Phi_{0}=4 \mathrm{~cm}^{3}\right)$ as a function of the LoS distance $R_{1}$. Performing a sweep of $\sigma_{h_{m i}}$ and $\Phi_{0}$, it is observed that the worse the road condition, the lower the roughness coefficient and, therefore, the lower the effect of the interference.

Table 1. Victim and interferer radar parameters. It is assumed that the radars transmit the same power, have similar radiation patterns and are vertically polarized. For the victim radar: $f_{s}=2.8571 \mathrm{MHz}$ and $B_{\mathrm{RX}}=7.5 \mathrm{MHz}$.

\begin{tabular}{|l|l|l|}
\hline Parameter Name & Victim & Interferer \\
\hline Carrier frequency $f_{c}$ & $76.5 \mathrm{GHz}$ & $76.41 \mathrm{GHz}$ \\
\hline Bandwidth $B$ & $1 \mathrm{GHz}$ & $1 \mathrm{GHz}$ \\
\hline Chirp duration $T_{c}$ & $179.2 \mu \mathrm{s}$ & $256 \mu \mathrm{s}$ \\
\hline Chirp repetition time $T_{r}$ & $350 \mu \mathrm{s}$ & $420.1 \mu \mathrm{s}$ \\
\hline Number of chirp ramps & 128 & 128 \\
\hline
\end{tabular}

\section{Interference Analysis}

Even though realistic traffic scenarios consider that the victim radar processes the superposition of backscattered signals of targets and signals transmitted by interferers, the following analysis focuses on the signal components related to interference. Considering just one interferer radar in the scenario, the superposition of the LoS component $s_{\mathrm{IF}_{1}}(t)$ and the two-path component $s_{\mathrm{IF}_{2}}(t)$ is given by

$$
\begin{array}{r}
s_{\mathrm{IF}}(t)=A_{1} \mathrm{e}^{\mathrm{j} \varphi_{\mathrm{IF} 1}}+A_{2} \mathrm{e}^{\mathrm{j} \varphi_{\mathrm{IF} 2}}=A_{1} \mathrm{e}^{\mathrm{j} \varphi_{\mathrm{IF} 1}}+A_{2} \mathrm{e}^{\mathrm{j} \varphi_{\mathrm{IF} 1}+\Delta \varphi} \\
\Delta \varphi(t)=2 \pi\left(-\frac{\mu_{I}}{c} t-\frac{f_{c_{I}}}{c}+\frac{\mu_{I}}{c^{2}} R_{1}+\frac{\mu_{I}}{2 c^{2}} \Delta R\right) \Delta R .
\end{array}
$$

Hence, the power of the superposed signals is calculated as

$$
\begin{aligned}
P & =\left|s_{\mathrm{IF}}(t)\right|^{2}=A_{1}^{2}+2 A_{1} A_{2} \cos (\Delta \varphi(t))+A_{2}^{2} \\
& =A_{1}^{2}-4 A_{1} A_{2} \sin ^{2}\left(\frac{\Delta \varphi(t)}{2}\right)+2 A_{1} A_{2}+A_{2}^{2} .
\end{aligned}
$$

Because of the sinusoidal dependency of the second term in (8), for a determined LoS distance $R_{1}$, the power of the superimposed signals takes a value within the interval

$$
\left[A_{1}^{2}+2 A_{1} A_{2}+A_{2}^{2}-4 A_{1} A_{2}, \quad A_{1}^{2}+2 A_{1} A_{2}+A_{2}^{2}\right] .
$$

The exact power value is given by the maximum of $\sin ^{2}(\Delta \varphi(t) / 2)$ evaluated in the time interval $\left[t_{I_{0}}, t_{I_{f}}\right]$.

A power analysis is performed by sweeping the LoS distance $R_{1}$. The simulated radar parameters of the victim and the interferer are presented in Table 1. Assuming that the sensors are synchronized, i.e., the transmission starts at the same time, the first interference takes place within the 6th victim chirp. For the subsequent analysis, this chirp is considered. Fig. 4 depicts the power of the received signal in the LoS $s_{\mathrm{IF}_{1}}(t)$ as well as the power of the superimposed signals $s_{\mathrm{IF}_{1}}(t)+s_{\mathrm{IF}_{2}}(t)$ for vertical and horizontal polarization. Furthermore, the upper and lower bounds mentioned in (9) are illustrated. As predicted before, horizontally polarized radars have a stronger effect, presenting deeper fadings and slightly higher peaks. From Fig. 4 it is also possible to recognize that

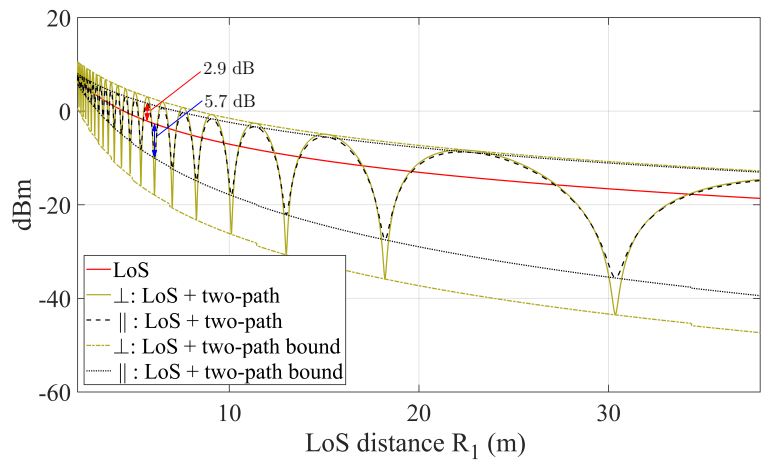

Fig. 4. Comparison of LoS signal component and LoS + two-path signal component for horizontally and vertically polarized radars assuming smooth asphalt. $R_{1} \in[0 \mathrm{~m}, 40 \mathrm{~m}]$. The labeled values correspond to the power difference produced by the two-path when the interferer is located at $R_{1}=4.53 \mathrm{~m}$ (constructive interference), and at $R_{1}=4.8 \mathrm{~m}$ (destructive interference).

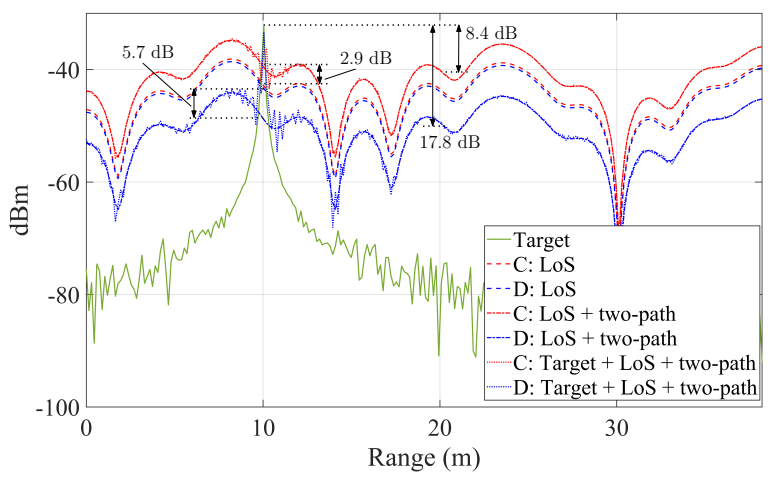

Fig. 5. Range profile of static target at $10 \mathrm{~m}$ and with $10 \mathrm{dBsm}$. Green: detected target without interference. Red: influence of the constructive interference (C) when interferer is located at $R_{1}=4.53 \mathrm{~m}$. Blue: influence of the destructive interference (D) when interferer is located at $R_{1}=4.8 \mathrm{~m}$.

the power of the superimposed signals oscillates around the power of the LoS component. Likewise, it is observed that the interval distance between two consecutive fadings or two consecutive peaks increases when $R_{1}$ rises.

Fig. 5 depicts the range profile when simulating a static target of $10 \mathrm{dBsm}$ at a distance of $10 \mathrm{~m}$ from the victim radar. It is possible to see the influence in the noise signal level due to the second path for the cases of constructive $\left(R_{1}=4.53 \mathrm{~m}\right)$ and destructive $\left(R_{1}=4.8 \mathrm{~m}\right)$ interference of the LoS and two-path signal components. In the first case, the signal level increases by $2.9 \mathrm{~dB}$ when adding the two-path component, while in the second case the signal level decreases by $5.7 \mathrm{~dB}$.

\section{Statistical Analysis and Measurement Results}

As stated before, the influence of the two-path component is given in (8). The power bounds given in (9) are not related to the chirp parameters of the involved radars, instead, they are determined by the scenario configuration and road properties. On the contrary, the phase shift of (7) is directly related to the interferer parameters $\mu_{I}$ and $f_{c_{I}}$. Random variations $\Delta \mu_{I}$ and $\Delta f_{c_{I}}$ will lead to the same curve depicted in Fig. 4, but shifted in the 


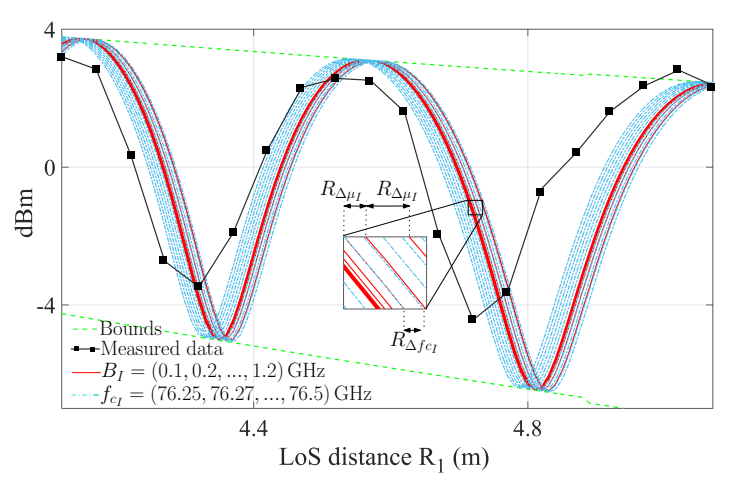

Fig. 6. Comparison of $\mathrm{LoS}+$ two-path signal component for vertically polarized radars and smooth asphalt. $R_{1} \in[4.1 \mathrm{~m}, 5.1 \mathrm{~m}]$. Red: variations in $\mu_{I}$ using $\Delta B_{I}=0.1 \mathrm{GHz}\left(R_{\Delta \mu_{I}}\right.$ is not constant). Blue: variations with $\Delta f_{c_{I}}=0.02 \mathrm{GHz}\left(R_{\Delta f c_{I}}\right.$ is constant). Black: Measured values.

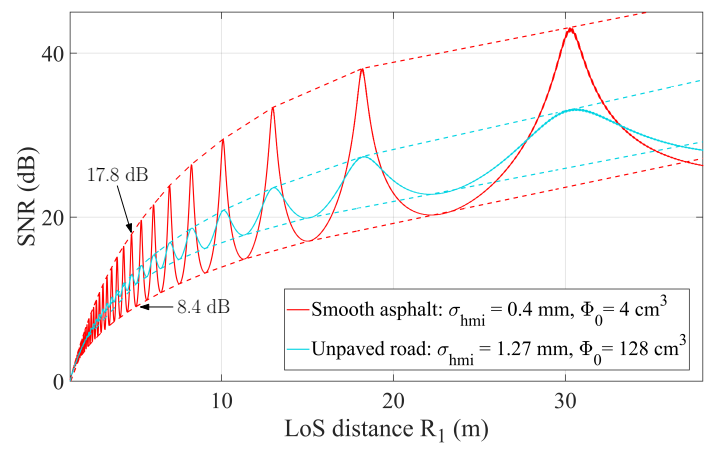

Fig. 7. SNR comparison for smooth asphalt (red) and an unpaved road (blue). The dotted lines represent the boundaries of the SNR variation while the solid lines depict the exact value for the simulated parameters. The SNR of the exemplary cases of Fig. 5 are labeled.

horizontal direction by distances $R_{\Delta f c_{I}}=\Delta R\left(\Delta f_{c_{I}} / f_{c_{V}}\right)$ and $R_{\Delta \mu_{I}}=\Delta R\left(\Delta \mu_{I} / f_{c_{V}}\right)\left(t+R_{1} / c+\Delta R / 2 c\right)$.

The influence of $\Delta \mu_{I}$ and $\Delta f_{c_{I}}$ is presented in Fig. 6. A uniform variation in $\Delta f_{c_{I}}$ leads to a uniform distance shifting of the curve. However, because of the time dependency of $R_{\Delta \mu_{I}}$, variations in $\Delta \mu_{I}$ do not produce a uniform distance shifting.

Fig. 7 depicts the signal-to-noise ratio (SNR) related to the target of Fig. 5 as a function of $R_{1}$ for smooth asphalt and for an unpaved road. It is observed that the SNR variations due to the influence of the second path are bounded and are determined by the road properties. In contrast to an unpaved road, a larger variation of SNR is seen for scenarios with smooth asphalt. As it was stated before, the influence of the two-path signal component is higher for better road conditions.

The performance of the proposed model is evaluated by taking measurements with two radars configured according to Table 1. The radars are placed in the anechoic chamber one in front of each other, and the distance between them is modified from $4 \mathrm{~m}$ to $5 \mathrm{~m}$, taking measurements for each $5 \mathrm{~cm}$. As presented in Fig. 8, a block of asphalt is placed in the middle in order to recreate the second path. The result is plotted in Fig. 6. The measurements are in a good agreement with the simulation, validating the proposed model.

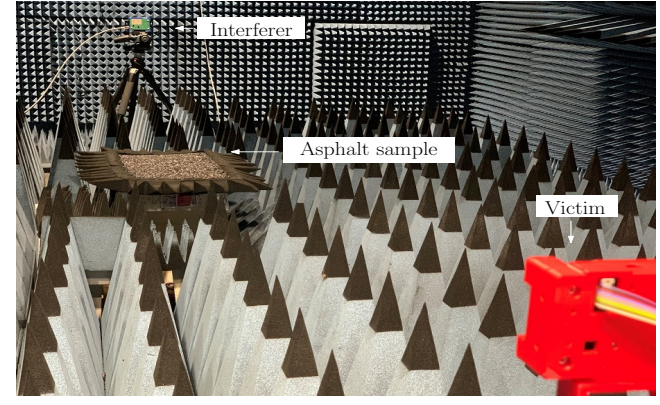

Fig. 8. Measurement setup.

\section{CONCLUSION}

A model used to simulate interference between automotive radars was presented and analyzed by considering the LoS and the two-path signal components of the interference. Effects of road material reflection properties and roughness were included in order to achieve a more realistic simulation environment. A lower effect of the interference was seen in vertically polarized radars. Moreover, when varying the distance between the victim and the interferer sensors, a bounded oscillating behavior of the received power was observed. It was found that the road material properties influence the maximum and minimum power bounds, while the interferer carrier frequency as well as the interferer frequency slope produce a shift in the fades and peaks of the power curve. Furthermore, the influence of interference in the SNR was studied showing that large variations can occur depending on the road material properties. Despite the fact that large variations in the SNR make impractical to model a particular scenario when the exact road properties are unknown, the overall behavior and effect of the interference can be simulated making use of the proposed approaches. The presented model is a fast and straightforward way to simulate the interference effects in complex traffic scenarios where multiple interfering sensors are present. It is an attractive approach to assess new mitigation strategies against interference.

\section{ACKNOWLEDGMENT}

The authors sincerely thank the German Federal Ministry of Education and Research for funding this research in the project IMIKO-Radar (German: Interferenzminimierung durch Kooperation bei Radarsensoren, grant: 16EMO0345).

\section{REFERENCES}

[1] F. Roos, J. Bechter, C. Knill, B. Schweizer, and C. Waldschmidt, "Radar sensors for autonomous driving: Modulation schemes and interference mitigation," IEEE Microwave Magazine, vol. 20, no. 9, pp. 58-72, Sep. 2019.

[2] M. Kunert, H. Meinel, C. Fischer, and M. Ahrhold, "Report on interference density increase by market penetration forecast," MOSARIM consortium, Tech. Rep., 2010.

[3] D. Oprisan and H. Rohling, "Analysis of Mutual Interference between Automotive Radar Systems," in 6th International Radar Symposium (IRS), 2005, pp. 83-90.

[4] R. Schneider, "Wave propagation modeling for vehicular-based radar imaging (in german)," Ph.D. dissertation, Universität Karlsruhe (TH), Germany, 1998. 